\title{
Excitation Functions of Stopping Power and Flow in Relativistic Heavy-Ion Collisions
}

\author{
Bao-An Li and C. M. Ko \\ Cyclotron Institute and Physics Department, Texas A\&M University, College Station, \\ TX 77843, USA
}

\begin{abstract}
Using a relativistic transport (ART) model, we study the stopping power, the formation of superdense hadronic matter as well as the strength of transverse and radial flow in central $\mathrm{Au}+\mathrm{Au}$ collisions at beam momentum from 2 to $12 \mathrm{GeV} / \mathrm{c}$ per nucleon. We find that complete stopping is achieved in the whole beam momentum range. In particular, the proton rapidity distribution scaled by the beam rapidity is independent of the beam momentum, and this is in agreement with the experimental findings. Also, a large volume of superdense hadronic matter with a local energy density exceeding that expected for the transition of a hadronic matter to the quark-gluon plasma is formed in collisions at beam momenta greater than $8 \mathrm{GeV} / \mathrm{c}$ per nucleon. Furthermore, it is found that the transverse flow in these collisions is sensitive to the nuclear equation of state and decreases with increasing beam momentum. On the other hand, the radial flow is insensitive to the equation of state, and its strength increases with beam momentum.
\end{abstract}

\section{INTRODUCTION}

Based on hydrodynamical models it has been predicted that the hadronic matter to quark-gluon plasma (QGP) phase transition may occur in heavy-ion collisions at incident energies between 2 and $10 \mathrm{GeV}$ per nucleon [ 1]. The formation of the quark-gluon plasma is expected to be accompanied also by the restoration of the chiral symmetry. To create the QGP requires that a large volume of high energy density matter is formed in the reactions. Whether this can be achieved in heavy ion collisions depends on the stopping power between the two colliding nuclei. It is therefore of interest to study how the stopping power and the volume of high density hadronic matter change with the incident energy. Also, collective nuclear flows are possible signatures for the formation of QGP. In Ref. [2], it has been shown using the hydrodynamical model that formation of the QGP in heavy ion collisions can lead to an abrupt change of the transverse and radial flows with respect to the incident energy. In this talk, we shall report results from our study on these questions using A Relativistic Transport (ART) model [3]. We note that experiments (e.g., E895, E866, E917, etc) are being carried out at Brookhaven's AGS to study $\mathrm{Au}+\mathrm{Au}$ collisions at incident beam momenta from 2 to $12 \mathrm{GeV} / \mathrm{c}$ per nucleon. Our study is thus useful in the search for the signatures of QGP formation and/or chiral symmetry restoration in these collisions. 


\section{THE EXCITATION FUNCTION OF NUCLEAR STOPPING POWER}

Nuclear stopping power can be inferred from the rapidity distribution of final nucleons. Complete stopping would lead to a rapidity distribution which peaks at the mid-rapidity, which has been observed in earlier experiments at AGS for central collisions of $\mathrm{Au}+\mathrm{Au}$ at an incident beam momentum of $12 \mathrm{GeV} / \mathrm{c}$ per nucleon [ [4. Such a proton distribution can be well reproduced by our ART model [3]. This model further predicts that complete stopping can also be achieved at lower beam momenta [ 5. It is, however, surprising to see that the scaled proton rapidity distribution, i.e., expressed in terms of $y / y_{\text {beam }}$ where $y_{\text {beam }}$ is the beam rapidity, from the model calculation at different beam energies all lie on a universal curve as shown in Fig. 1. This simple scaling is in quantitative agreement with what has been found by Harris [7] based on the analysis of experimental data from heavy ion collisions at incident energies from 0.25 to $160 \mathrm{GeV}$ per nucleon. This scaling behavior may be related to the Fermi-Landau scaling [ 8] of the pion multiplicity in a nucleon-nucleon collision, i.e., it increases more or less linearly with the available centerof-mass energy. Since the nucleon-nucleon inelastic cross section used in the ART model has the Fermi-Landau scaling, the pion multiplicity per participant in heavy ion collisions is also found to follow a similar dependence on the center-of-mass energy [5]. It is thus of interest to study in the ART model how the pion multiplicity and the scaled proton rapidity distribution are modified when the Fermi-Landau scaling is violated in the input cross sections.

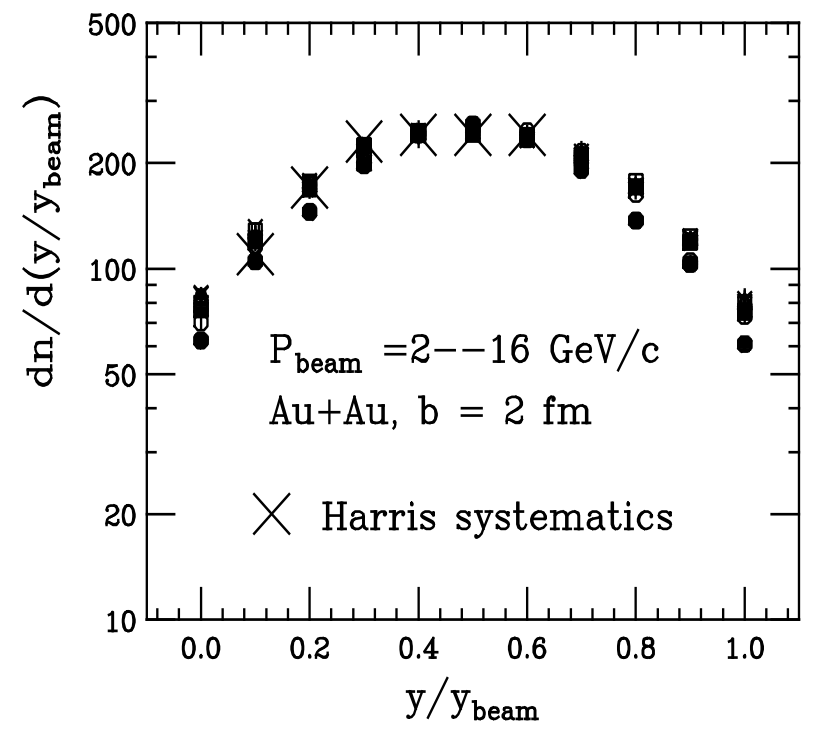

Figure 1. The scaled proton rapidity distribution from the reaction of $\mathrm{Au}+\mathrm{Au}$ at an impact parameter of $2 \mathrm{fm}$ and beam momentum of 2 to $16 \mathrm{GeV} / \mathrm{c}$ per nucleon. The large crosses are from Ref. [7]. 


\section{THE EXCITATION FUNCTION OF SUPERDENSE HADRONIC MAT- TER FORMATION}

The complete stopping of two colliding nuclei in central $\mathrm{Au}+\mathrm{Au}$ reactions at $P_{\mathrm{beam}} / A=$ 2 to $12 \mathrm{GeV}$ implies that high energy density matter is created in the collision. As a useful guidance for the search of QGP at the AGS, we show in Fig. 2 the time evolution of the volume of hadronic matter where the local energy density is higher than the estimated transition energy density of about $2.5 \mathrm{GeV} / \mathrm{fm}^{3}$ between the hadronic matter and the quark-gluon plasma [ 9]. Our results indicate that the transition to QGP can already occur at a beam momentum of about $8 \mathrm{GeV} / \mathrm{c}$. With increasing beam momentum, the volume of such a high energy density matter becomes more significantly larger.

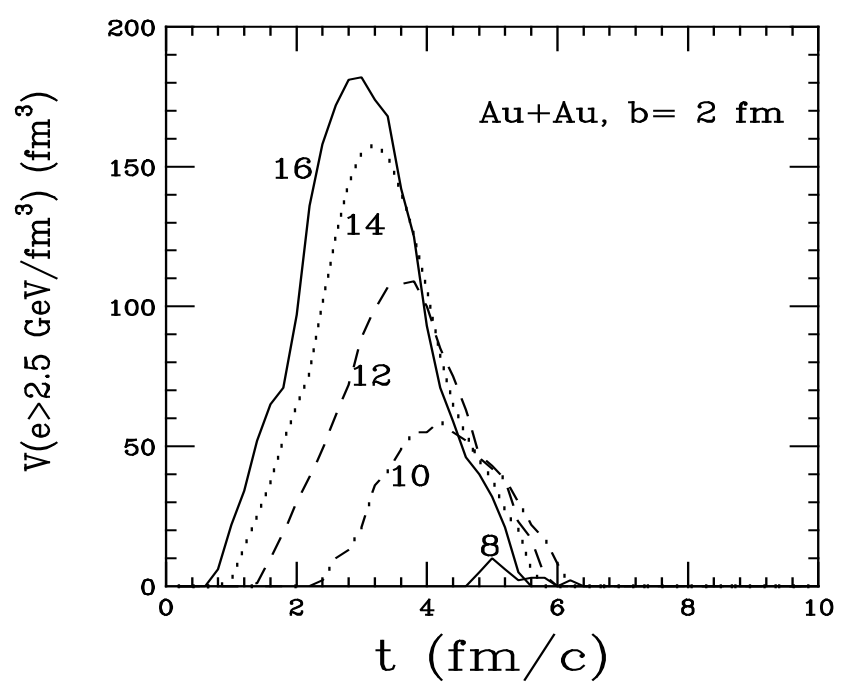

Figure. 2. The volume of hadronic matter with local energy density higher than 2.5 $\mathrm{GeV} / \mathrm{fm}^{3}$ as a function of time and beam momentum for the reaction of $\mathrm{Au}+\mathrm{Au}$ at an impact parameter of $2 \mathrm{fm}$ using the cascade mode of ART.

The maximum compression reached in a heavy-ion collision can also be determined by solving the Rankine-Hugoniot (RH) equation [ 10]. In the relativistic hydrodynamical model [ 11], the maximum baryon density $\rho$ is found to satisfy the following equation,

$f(\rho)(n-\gamma)-e_{0} n\left(B \gamma^{2}+\gamma^{2}-1-B \gamma n\right)=0$

where $n \equiv \rho / \rho_{0}$ and $e_{0}$ is the energy density of the nuclear matter in the front of the shock wave; $\gamma$ is the Lorentz factor of the beam energy in the nucleus-nucleus center of mass frame; $B$ is the ratio of the thermal pressure to the energy density. We find that up to the beam momentum of about $10 \mathrm{GeV} / \mathrm{c}$ per nucleon a value of $B=2 / 3$ corresponding to a non-relativistic gas can best describe our results from the transport model. In the 
above, $f(\rho)$ is defined as

$f(\rho) \equiv \rho^{2} d\left(\frac{e_{0}(\rho)}{\rho}\right) / d \rho-B e_{0}(\rho)$,

where $e_{0}(\rho)$ is the energy density at zero temperature. For a soft equation of state as used in the transport model, we have

$e_{0}(\rho) / \rho=m_{N}+\frac{a}{2} \frac{\rho}{\rho_{0}}+\frac{b}{1+\sigma}\left(\frac{\rho}{\rho_{0}}\right)^{\sigma}+\frac{3}{5} E_{f}\left(\frac{\rho}{\rho_{0}}\right)^{2 / 3}$

and

$f(\rho)=\rho_{0}\left(-\frac{2}{3} m_{N} n+\frac{a}{6} n^{2}+\frac{b(\sigma-2 / 3)}{1+\sigma} n^{\sigma+1}\right)$,

where $a=-358.1 \mathrm{MeV}, b=304.8 \mathrm{MeV}, \sigma=7 / 6, E_{f}$ is the Fermi energy in the normal nuclear matter, and $m_{N}$ is the nucleon mass. In the case of a free nucleon gas, i.e., $a=b=0$, one obtains the following analytical solution for the maximum baryon density [12],

$n=\frac{5 \gamma^{2}-2 \gamma-3}{2(\gamma-1)}=\frac{1}{2}(5 \gamma+3)$.

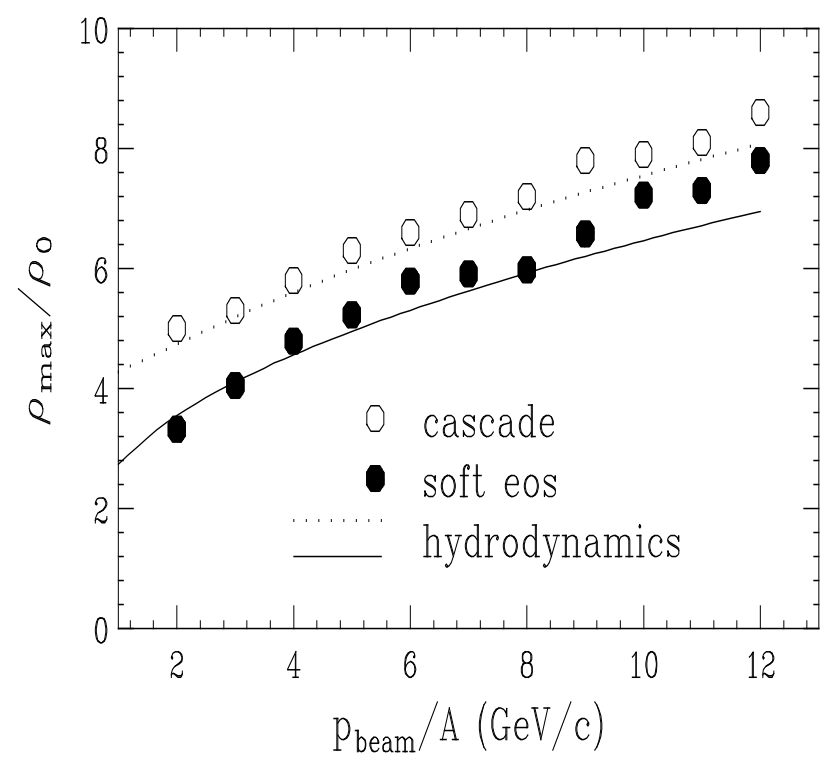

Figure 3. The beam momentum dependence of the maximum baryon density for the reaction of $\mathrm{Au}+\mathrm{Au}$ at an impact parameter of $2 \mathrm{fm}$. The dotted and solid lines are predictions of the relativistic hydrodynamics.

The maximum baryon density obtained from Eq. (5) is shown by the dotted line in Fig. 3. The solid line is the result from solving numerically Eq. (1) using an iterative method 
and a soft equation of state corresponding to a compressibility of $210 \mathrm{MeV}$. Results from the cascade model and the transport model with the soft equation of state are shown by the open and the filled circles, respectively. It is amazing that predictions from the two models agree very well in both cases of free and interacting nucleon gas, especially at beam momenta below $9 \mathrm{GeV} / \mathrm{c}$ per nucleon. Small discrepancies between the two model predictions appear when the beam momentum is higher than about $10 \mathrm{GeV} / \mathrm{c}$ per nucleon. This is, however, expected as the system under study deviates from a non-relativistic gas at increasingly higher energies, and consequently the parameter $B$ should be smaller than $2 / 3$. Also, the conditions for the applicability of the hydrodynamical approach, such as local thermal equilibrium, may not be realized in the transport model when the incident energy is high.

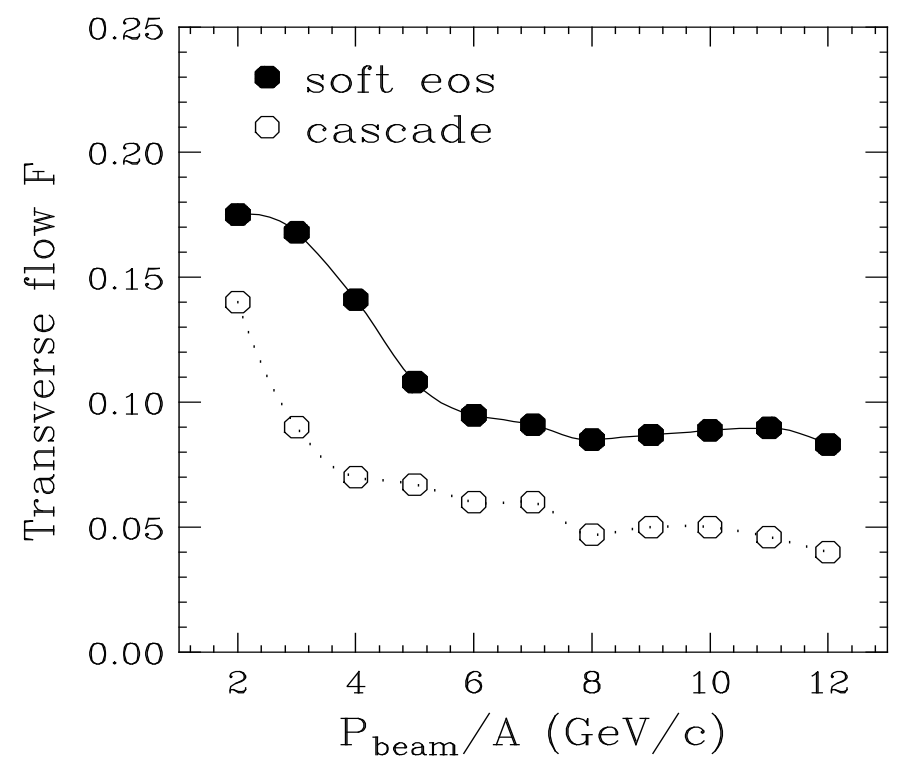

Figure 4. The transverse flow parameter as a function of beam momentum for the reaction of $\mathrm{Au}+\mathrm{Au}$ at an impact parameter of $2 \mathrm{fm}$.

\section{EXCITATION FUNCTIONS OF TRANSVERSE AND RADIAL FLOWS}

To study the transverse flow we use the standard method of Danielewicz and Odyniec [ 13], i.e., we analyze the average transverse momentum in the reaction plane as a function of rapidity. We also extract the flow parameter $F \equiv\left(d p_{x} / d y\right)_{y=0}$ and study its dependence on the beam energy. Results of this analysis from both the cascade and the transport model with the soft equation of state are shown in Fig. 3 for the reaction of $\mathrm{Au}+\mathrm{Au}$ at an impact parameter of $2 \mathrm{fm}$. Two interesting features are seen in these results. First, in both cases the transverse flow parameter $F$ shows a strong decrease as the beam momentum increases from 2 to about $7 \mathrm{GeV} / \mathrm{c}$ per nucleon. Above these momenta, it becomes more or less a constant. Secondly, at all energies the predicted flow parameter from the cascade is about a factor of two smaller than that using the soft equation of state. These effects 
can be understood schematically by considering the maximum flow parameter $F_{\max }$ as a function of beam momentum, which is approximately given by

$F_{\max } \approx\left(\left\langle\Delta p_{x}\right\rangle_{c}\right) / y_{\mathrm{cm}}+\left(\int_{0}^{t_{r}} F_{x} d t\right) / y_{\mathrm{cm}}$

In the above, $\left\langle\Delta p_{x}\right\rangle_{c}$ is the net transverse momentum generated by nucleon-nucleon collisions; $F_{x}$ is the nuclear force acting on nucleons along the $+x$ direction in the reaction plane; and $t_{r} \propto 1 / \gamma$ is the reaction time. In pure cascade calculations, the flow is due to nucleon-nucleon collisions, which decreases with increasing beam energy as a result of a rapidly decreasing reaction time $t_{r}$ during which the thermal pressure creates a sideward deflection in the reaction plane. Including mean-field potential in the model leads to the second term, which depends not only on the reaction time but also on the density gradient of the compressed matter. Although the latter increases with increasing beam energy, which gives rise to a stronger nuclear force at high energies, the shorter reaction time at higher energies reduces its effect. One thus expects that the second term in Eq. (6) contributes more or less equally at all energies, and this is indeed seen from the near constant difference between the two results shown in the figure.

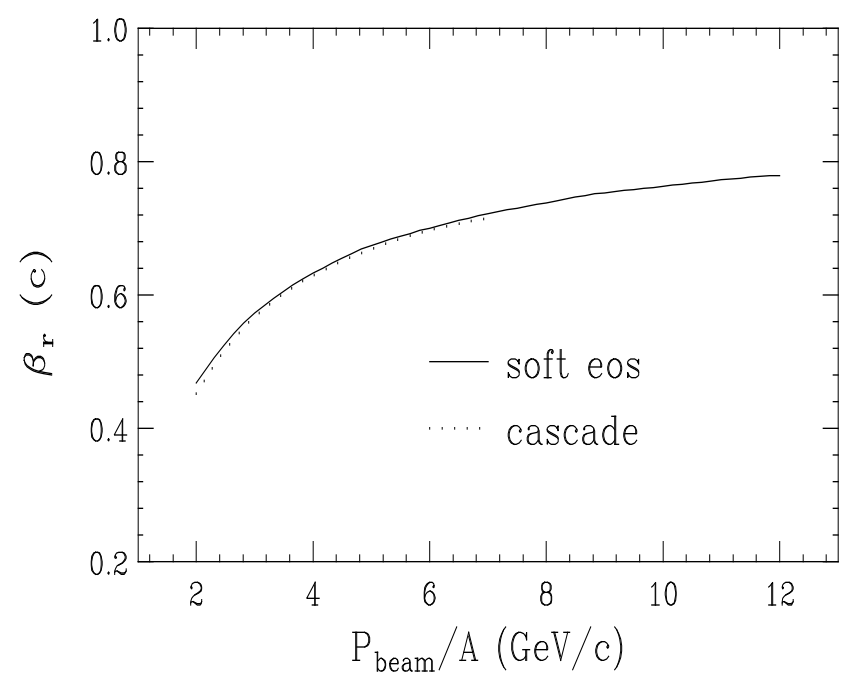

Figure 5. The average radial flow velocity as a function of beam momentum for the reaction of $\mathrm{Au}+\mathrm{Au}$ at an impact parameter of $2 \mathrm{fm}$.

The radial flow is characterized by the average radial flow velocity $\beta_{r}$ defined by

$\beta_{r} \equiv \frac{1}{N} \sum_{i}^{N} \frac{\overrightarrow{p_{i}}}{E_{i}} \cdot \frac{\overrightarrow{r_{i}}}{r_{i}}$,

where the summation is over all test particles in the system. The value of $\beta_{r}$ at $\mathrm{t}=20 \mathrm{fm} / \mathrm{c}$ is shown in Fig. 5 for both the cascade and the transport model with the soft equation 
of state. We see that the radial flow velocity increases quickly with the beam momentum and reaches a constant value of about $0.78 \mathrm{c}$. The similar results from these two cases further indicate that the radial flow is mainly determined by the thermal pressure rather than the potential pressure. Since the thermal pressure increases with increasing beam energy, the radial flow also becomes larger at higher energies.

\section{SUMMARY}

Based on the relativistic transport model (ART 1.0) we have studied the excitation functions of stopping, compression and collective flow in central $\mathrm{Au}+\mathrm{Au}$ reactions at beam momenta from 2 to $12 \mathrm{GeV} / \mathrm{c}$ per nucleon. We find that complete stopping is reached in the whole beam momentum range and the proton rapidity distribution scales with the beam rapidity. A large volume of superdense hadronic matter is seen to form in these reactions. The calculated maximum baryon and energy densities are in good agreement with predictions of the relativistic hydrodynamics which assumes the formation of a shock wave in heavy-ion collisions. The nucleon transverse flow turns out to be sensitive to the equation of state in this beam momentum range, and its strength is found to decrease with increasing beam momentum. On the other hand, the radial flow is insensitive to the nuclear equation of state and increases with the beam momentum. In our model, neither the quark-gluon plasma nor the chiral restored phase is included. The results presented here are based on purely hadronic dynamics. They are, however, useful in the sense that deviations of experimental observations from our predictions might signal the existence of new phenomena that are related to chiral symmetry restoration and/or quark-gluon-plasma formation in heavy-ion collisions at Brookhaven's AGS.

This work was supported in part by the NSF Grant No. PHY-9509266.

\section{REFERENCES}

1. M. Gyulassy, Nucl. Phys. A590, 431c (1995).

2. D.H. Rischke, in Proc. of Heavy-Ion Physics at the AGS, edited by C.A. Pruneau et al., p. 138 (1996).

3. B.A. Li and C.M. Ko, Phys. Rev. C52, 2037 (1995); ibid, C53, R22 (1996).

4. F. Videbaek, Nucl. Phys. A590, 249c (1995).

5. B.A. Li and C.M. Ko, Nucl. Phys. A601, 457 (1996).

6. B.A. Li and W. Bauer, Phys. Lett. B254, 335 (1991); Phys. Rev. C44, 450 (1991).

7. J.W. Harris, in Advances in Nuclear Dynamics 2, Eds. W. Bauer and G.D. Westfall, Plenum Press, New York, 1996, page 401.

8. E. Fermi, Prog. Theor. Phys. V5, 750 (1950); L.D. Landau, Izv. Akad. Nauk SSSR, Ser. Fiz. V17, 51 (1953).

9. C.Y. Wong, Introduction to High Energy Heavy-Ion Collisions (World Scientific, Singapore, 1994).

10. A.H. Taub, Phys. Rev. 74, 328 (1948).

11. R.B. Clare and D. Strottman, Phys. Rep. 141, 177 (1986).

12. A.A. Amsden, F.H. Harlow and J.R. Nix, Phys. Rev. C15, 2059 (1977).

13. P. Danielewicz and G. Odyniec, Phys. Lett. B157, 146 (1985). 